\title{
Fish oil supplementation associated with decreased cellular degeneration and increased cellular proliferation 6 weeks after middle cerebral artery occlusion in the rat
}

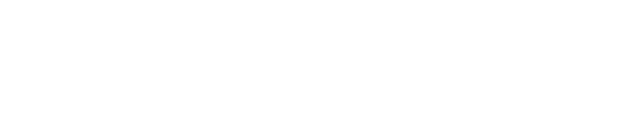

\section{Michaela C Pascoe' \\ David W Howells ${ }^{2}$ \\ David P Crewther ${ }^{1}$ \\ Leeanne M Carey ${ }^{2,3}$ \\ Sheila G Crewther ${ }^{4}$}

'Brain Sciences Institute, Swinburne University, ${ }^{2}$ Florey Institute of Neuroscience and Mental Health, University of Melbourne, ${ }^{3}$ Department of Occupational Therapy, School of Allied Health La Trobe University, ${ }^{4}$ School of Psychological Science, La Trobe University, Melbourne, VIC, Australia
Correspondence: Michaela C Pascoe Institute of Neuroscience and Physiology, Dept of Clinical Neuroscience and Rehabilitation, Sahlgrenska Academy at University of Gothenburg, Box 440 SE-405 30 Gothenburg, Sweden $\mathrm{Tel}+46317863147$

Email michaela.pascoe@neuro.gu.se

\begin{abstract}
Anti-inflammatory long-chain omega-3 polyunsaturated fatty acids (n-3-LC-PUFAs) are both neuroprotective and have antidepressive effects. However the influence of dietary supplemented n-3-LC-PUFAs on inflammation-related cell death and proliferation after middle cerebral artery occlusion (MCAo)-induced stroke is unknown. We have previously demonstrated that anxiety-like and hyperactive locomotor behaviors are reduced in n-3-LC-PUFA-fed MCAo animals. Thus in the present study, male hooded Wistar rats were exposed to MCAo or sham surgeries and examined behaviorally 6 weeks later, prior to euthanasia and examination of lesion size, cell death and proliferation in the dentate gyrus, cornu ammonis region of the hippocampus of the ipsilesional hemispheres, and the thalamus of the ipsilesional and contralesional hemispheres. Markers of cell genesis and cell degeneration in the hippocampus or thalamus of the ipsilesional hemisphere did not differ between surgery and diet groups 6 weeks post MCAo. Dietary supplementation with n-3-LC-PUFA decreased cell degeneration and increased cell proliferation in the thalamic region of the contralesional hemisphere. MCAo-associated cell degeneration in the hippocampus and thalamus positively correlated with anxiety-like and hyperactive locomotor behaviors previously reported in these animals. These results suggest that anti-inflammatory n-3-LC-PUFA supplementation appears to have cellular protective effects after MCAo in the rat, which may affect behavioral outcomes.

Keywords: apoptosis, polyunsaturated fatty acids, contralesional hemisphere, stroke, anxiety, depression
\end{abstract}

\section{Introduction}

Ischemic stroke is experienced by 15 million people worldwide each year. ${ }^{1}$ Stroke has a personal, public health, and financial burden, and thus, it is important to achieve the best possible outcomes. Ischemia is associated with central nervous system cell death and proliferation, which play a significant role in functional recovery and outcome. ${ }^{2-4}$ Ischemia-related cell death in animals is associated with the presentation of behaviors believed to model disorders reported by stroke survivors, ${ }^{4-11}$ including hyperactive delirium (increased motor activity with agitated behavior, restlessness, agitation, and hypervigilance, and often hallucinations and delusions), ${ }^{7,12}$ anxiety ${ }^{13,14}$ and clinical depression, ${ }^{15,16}$ all of which negatively influence functional recovery. ${ }^{15}$

Long-chain omega-3 polyunsaturated fatty acids (n-3-LC-PUFAs) are dietary lipids found in high concentrations in cell membranes, most abundantly in the central nervous system. ${ }^{17}$ At optimal doses, n-3-LC-PUFAs derived from dietary fish oil have been shown to have anti-inflammatory effects. ${ }^{18}$ n-3-LC-PUFAs have been 
associated with reduced infarct size 3 days after middle cerebral artery (MCA) occlusion (MCAo) in cats, following diet supplementation for 24 days prior to surgery. ${ }^{19}$ Rapeseed oil, rich in omega-3 alpha-linolenic acid (ALA), is associated with reduced lipid peroxidation, infarct size, and mortality, when diet is supplemented 4-6 weeks prior to ischemia. ${ }^{20}$ In rodents, three sequential injections of ALA has also been shown to promote neural plasticity 10 days post-MCAo surgery. ${ }^{21}$ Gavage feeding of n-3-LC-PUFA for 2 weeks prior to ischemic surgery ${ }^{22}$ and for 8 weeks prior to hippocampal injection of scopolamine, reduces oxidative stress and apoptosis. ${ }^{23}$ To our knowledge, the influence of longer-term poststroke n-3-LC-PUFA dietary supplementation on cellular degeneration and proliferation has not been investigated in either animal models or clinical populations. We have previously demonstrated that 6 weeks of dietary supplementation with n-3-LC-PUFA, which best models the method of human consumption, is associated with reduced locomotor hyperactivity and anxiety-like behavior in the rat. ${ }^{24}$ We hypothesize that these behavioral changes result, in part, from the cellular protective effects of n-3-LC-PUFA supplementation, after inflammatory stroke or sham surgery.

In rodent models, MCAo-induced cortical infarction produces secondary cellular damage in the thalamic region of the ipsilesional hemisphere for at least 9 months postsurgery. ${ }^{25-28}$ The contralateral hemisphere is similarly involved in structural remodeling. ${ }^{29,30}$ The thalamus is essential for information processes necessary for sensory and motor control. ${ }^{31-35}$ Accordingly, hyperactive delirium after stroke is associated with cellular damage in the thalamus in clinical populations. ${ }^{7-9,12}$ In animal models, hyperactive locomotion is associated with cellular damage in the cornu ammonis (CA1) region of the ipsilesional hemisphere. ${ }^{4,10}$ Hippocampus cells are considered to be particularly vulnerable to cellular degeneration after cerebral ischemia. ${ }^{36,37}$ Additionally, the hippocampus is involved in adult neurogenesis, and ischemia-induced cellular proliferation has been widely demonstrated to occur in the hippocampus..$^{38}$ In the gerbil model, cell proliferation is seen particularly in the hippocampus ipsilateral to the injury for up to 2 months poststroke. ${ }^{39}$ Finally, a decrease in neurogenesis and an increase in neurodegeneration, in the CA1 and dentate gyrus (DG) of the hippocampus, are widely implicated in the etiology of depressive and anxiety-like disorders., 5

Thus, to better understand the behavioral effects of the balance between neurodegenerative and neurogenic processes in the rodent model 6 weeks post-transient ischemia, we immunohistochemically examined tissue prepared from MCAooperated and sham control animals, some of whom had been fed a n-3-LC-PUFA diet. Using caspase-3 and Ki-67 protein, we aimed to establish whether persistent (6 weeks following surgery) stroke-related cellular proliferation, in general, would correlate with the presentation of depressive/anxiety-like and locomotor behaviors, or be influenced by dietary intervention. Caspase-3 has been widely employed as a marker of cell death after traumatic brain injury ${ }^{40}$ and MCAo. ${ }^{41}$ Caspase activation has been observed in rodent brain tissue from as early as a few hours postsurgery to as late as several weeks poststroke. ${ }^{37,41-44}$ The Ki-67 protein is a reliable marker present during all active phases of the cell cycle and is commonly employed to detect cellular proliferation. ${ }^{45}$ Indeed, previous authors have demonstrated increased Ki-67 expression in the dentate subgranular zone as late as 7 weeks post-MCAo in rodent models. ${ }^{38}$

In the present study, we hypothesized that MCAo surgery compared with sham surgery would be associated with increased caspase- 3 and Ki-67. We hypothesized that 6 weeks of dietary supplementation with n-3-LC-PUFA would also influence the expression of caspase- 3 and $\mathrm{Ki}-67$, in the thalamus of the ipsilateral and contralateral hemisphere, and the CA1 and DG of the hippocampus of the ipsilateral hemisphere. We finally hypothesized that hyperactive locomotor and anxiety-like behaviors, previously reported in these animals ${ }^{24}$ would be correlated with caspase-3 and Ki-67 expression.

\section{Materials and methods Animals and dietary regime}

The experimental protocol required 48 animals; however, eleven died immediately following withdrawal of the suture thread during the surgical procedure. A further 16 rats were culled due to stroke-induced symptoms deemed too large for the animal to survive. These animals were replaced, and thus, the total number of animals used in the present study was 75 (Laboratory Animal Services, The University of Adelaide, Adelaide, Australia), with body weight $=337 \mathrm{~g}$ (standard deviation $[\mathrm{SD}]=36.5 \mathrm{~g}$ ) and age 12-14 weeks, randomly allocated to receive basal (AIN93G; Specialty Feeds, Glen Forest, Australia) diet or high-n-3-LC-PUFA (eicosapentaenoic acid [EPA] 20:5 n3 5\% of total free fatty acid [FFA], docosahexaenoic acid [DHA] 22:6 n3 23.8\% of total fatty acids) (SF09-109 5\% Fat High N3 Modified Rodent Diet; Specialty Feeds) diet previously demonstrated to reduce oxidative stress in the rat model, ${ }^{46}$ and either sham or MCAo surgery condition; thus there were four experimental groups: n-3-LC-PUFA diet plus MCAo ( $\mathrm{n}=11)$; basal diet plus MCAo (n=13); n-3-LC-PUFA diet plus sham surgery $(\mathrm{n}=12)$; and basal diet plus sham surgery $(\mathrm{n}=12)$. Rats were housed with a $12: 12$ hours light-dark cycle at $21^{\circ} \mathrm{C} \pm 2^{\circ} \mathrm{C}$ and acclimated to diet and housing the week prior to surgery. Dietary supplementation continued until the sixth 
week postsurgery, when the animals were sacrificed for tissue collection. Food and water was available ad libitum. Procedures were approved by the Austin Health Research Ethics Unit (approval number A2010/03865) and conducted in accordance with the Australian Code of Practice for the Use of Animals for Scientific Purposes. ${ }^{47}$

\section{Middle cerebral artery occlusion}

This model has been described previously. ${ }^{24}$ Briefly, rats were anesthetized (isoflurane; Baxter International, Inc., Deerfield, IL, USA) (5\% in oxygen, maintained $2 \%$ in oxygen) and atropine (intraperitoneal route) $(0.2 \mathrm{~mL} 600 \mu \mathrm{g} / \mathrm{mL}$; Pfizer, Inc., New York, NY, USA) administered. Blood flow was measured using laser doppler ( $1 \mathrm{~mm}$ caudal and $5 \mathrm{~mm}$ lateral to the bregma). Branch arteries and the external carotid artery were ligated, and an incision was made in the right external carotid artery through a surgical incision in the neck. A $0.4 \mathrm{~mm}$ silicone-tipped suture was inserted into the internal carotid artery (18 $\mathrm{mm}$ from carotid bifurcation). The MCAo occlusion period was 90 minutes. The occluding suture was removed into the external carotid stump for reperfusion. Incisions were closed with silk sutures. Sham-operated animals underwent procedures identical to MCAo animals, excluding thread insertion into the MCA.

\section{Behavioral correlations}

We previously tested rats on the battery of behavioral tests described below at 6 weeks poststroke, ${ }^{24}$ and thus, behavioral results are not reported in the present study. In the present study, we report correlations between the previously reported behavioral outcomes with histological and whole-brain protein analysis. The methodologies have been previously described. ${ }^{24}$ Briefly, hyperactive locomotor behaviors were studied using a free-exploration test. "Locomotor hyperactivity" was defined as a high number of emergences from the "hide" box into the open field and the duration of time spent moving in the open field arena. "Anxiety-like behavior" was defined as the percentage of emergence time spent along the outer wall of the open field arena compared with the time spent in the center of the open field. Additionally, novel-object exploration while in a familiar environment was interpreted to indicate approach versus avoidance behavior.

\section{Tissue collection}

Animals were anaesthetized and transcardially perfused (Peri-Star Pro 4-channel, high rate pump; World Precision Instruments, Inc., Sarasota, FL, USA) with saline (1.8\%) and paraformaldehyde (4\%). Tissue was postfixed in paraformaldehyde ( 24 hours) followed by $30 \%$ sucrose and then processed using a closed linear tissue processing system (Tissue Processing Center TPC 15 Duo/Trio; MEDITE GmbH, Burgdorf, Germany). Sections were embedded in molten paraffin, sectioned $(7 \mu \mathrm{m}$, room temperature [RT]) using a rotary microtome, and attached to silane-coated slides (Mikro-Glass; Grale Scientific Pty Ltd., Ringwood, Australia). For the Western blot analysis, animals were anaesthetized, and brains were collected via decapitation using a guillotine, placed immediately into liquid nitrogen, and stored at $-80^{\circ} \mathrm{C}$ until analysis.

\section{Cellular markers}

We examined the relationship between n-3-LC-PUFA supplementation and the number of caspase-3 (Polyclonal Cleaved Caspase-3 [Asp175] Antibody; Genesearch, Arundel, Australia) and Ki-67-like immunoreactivity (Ki-67-ir)-labeled cells (AntiKi-67 antibody [SP6] - Monoclonal Proliferation Marker; Sapphire Bioscience, San Diego, CA, USA), as a general marker of cellular proliferation. For the Western blot analysis, cell death expression in whole-brain tissue was defined as the total mean protein expression of caspase-3, normalized to the commonly used glyceraldehyde 3-phosphate dehydrogenase (GAPDH) (Sigma-Aldrich Corp, St Louis, MO, USA). ${ }^{48,49}$

\section{Cell counting}

Photomicrographs were taken using an Eclipse 90i upright microscope (Nikon Corporation, Tokyo, Japan) and a Nikon DS-Fi1 digital camera (Nikon) (1,280×960 pixels). A standard stereotaxic rat brain atlas ${ }^{50}$ was used to identify relevant anatomical regions. Ki-67-ir appeared as an orange/brown precipitate localized to the cell nuclei. Caspase-3-like immunoreactivity (caspase-3-ir) appeared as an orange/brown precipitate localized to the cell nuclei as well as dense plaque-like deposits. Cell number was determined using NIS Elements software (Version 3.2; Nikon). Cell counting within photographs was restricted to a predefined area $(1,064.7 \times 521.5$ $\mu \mathrm{m})$. Two photographs were taken of the CA1 region and DG of each animal, and an average cell number was derived from these. To allow for assessment of the ipsilesional and contralesional thalamic regions, photographs in each of the hemispheres were taken of the mediodorsal thalamic nucleus, posterior thalamic nuclear group, ventromedial thalamic nucleus, ventral posterolateral thalamic nucleus, and ventral posteromedial thalamic nucleus, as outlined in Figure 1, and an average cell number was derived from these. To allow for assessment of the ipsilesional hippocampus, photographs were taken of the CA1 field of the hippocampus and DG. Cell number was determined using a threshold parameter, where only pixels with a given range of color, identified as representing caspase-3-ir and $\mathrm{Ki}-67$-ir cells, were included 


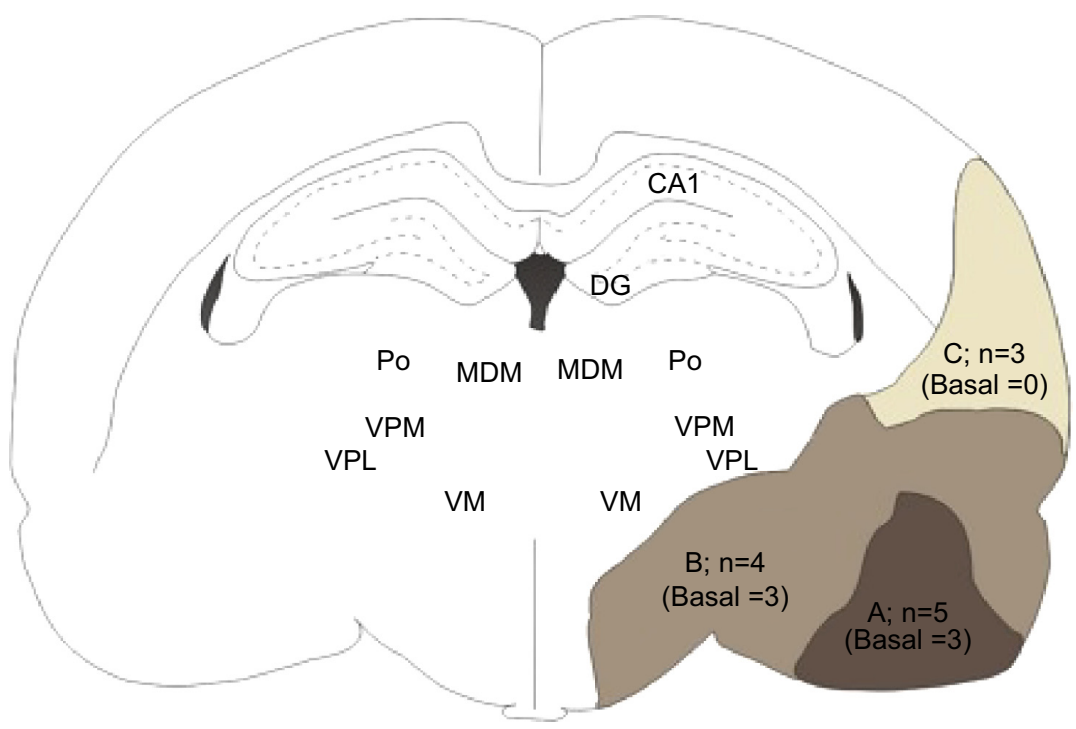

Figure I Line drawing showing infarct area and brain regions selected for analysis of caspase-3-like and Ki-67-like immunoreactivity, 6 weeks after middle cerebral artery occlusion.

Notes: Shaded areas show the extent of infarct. $A=$ area of atrophy in animals with smaller infarcts; $B=$ area of atrophy additional to area $A$, in animals with medium infarcts; $C=$ area of atrophy additional to areas $A$ and $B$, in animals with large infarcts. Basal = basal diet-fed rats.

Abbreviations: CAI, cornu ammonis area of the hippocampus; DG, dentate gyrus; MDM, mediodorsal thalamic nucleus; Po, posterior thalamic nuclear group; VM, ventromedial thalamic nucleus; VPL, ventral posterolateral thalamic nucleus; VPM, ventral posteromedial thalamic nucleus.

in the analysis. An area restriction of $40 \mu \mathrm{m}^{2}$ was set to ensure that dark-stained objects too small to be cell nuclei were not counted in the case of Ki-67-ir cells. In the case of caspase-3, an area restriction was not set, to ensure that the smaller caspase-3-ir pigmentation and plaques were detected. Automated counting was significantly correlated with manual counting $\left(r^{2}=0.91\right)$. Results were analyzed by an observer blind to experimental treatment condition.

\section{Western blot}

Frozen tissue was pulverized, lysed $\left(5\right.$ minutes, $\left.95^{\circ} \mathrm{C}\right)$ in Laemmli buffer (63 mM). Samples were centrifuged, and the proteins in the supernatant were loaded into 4\%-20\% precast gels (Major Sciences, Saratoga, CA, USA) in sodium dodecyl sulfate (SDS) buffer (Lonza Group, Basel, Switzerland) and subsequently transferred to polyvinylidene difluoride (PVDF) membrane. Membranes were developed with cleaved caspase-3, 1:500 or GAPDH, 1:5,000 $\left(4^{\circ} \mathrm{C}\right)$ and detected using enhanced chemiluminescence (ECL) mix (GE Healthcare, Little Chalfont, UK). A given band of intensity was calculated as the sum of the values of the pixels in the image.

\section{Histomorphologic evaluation}

Sections were blocked (60 minutes) in hydrogen peroxide (0.3\%; Chem-Supply Pty, Ltd., Gillman, Australia) followed by normal horse serum (Invitrogen ${ }^{\circledR} 10 \%$; Life Technologies, Carlsbad, CA, USA). Tissue was then incubated overnight with the primary antibody, (Anti-Ki-67, 1:1,000; or caspase- $3,1: 1,000)\left(4^{\circ} \mathrm{C}\right)$ followed by the secondary antibody (Biotinylated Horse Anti-Rabbit IgG Antibody; Abacus ALS, Meadowbrook Australia) (60 minutes RT). Finally sections were incubated with avidin biotin complex solution (ABACUS ALS) (60 minutes RT), and 3-3'diaminobenzidine tetrahydrochloride (1\%; Sigma-Aldrich Corp) (20 minutes RT) with addition of $\mathrm{H}_{2} \mathrm{O}_{2}(0.01 \%, 15$ minutes RT). Tissue was background-stained using hematoxylin and eosin. Infarct volumes were calculated according to Cavalieri's principle, according to the equation:

$$
\mathrm{V}=\sum \mathrm{A} \times \mathrm{P} \times \mathrm{T}^{51}
$$

where $\mathrm{V}$ is the total volume, $\Sigma \mathrm{A}$ is the sum of areas measured, $\mathrm{P}$ is the inverse of the sampling fraction of the section, and $\mathrm{T}$ is the section thickness.

Areas of tissue damage were analyzed using Stereo Investigator $^{\circledR}$ Version 6 software.

\section{Data analysis}

Statistical analysis was performed using SPS Statistics (version 20 for Windows; IBM Corp, Armonk, NY, USA). Outliers were screened using box plots. Sphericity was checked using Mauchly's test of sphericity, and homogeneity of variance was checked using Levine's test of equality of error variance. Normality was assessed using Q-Q plots and 
histograms. Two-way analysis of variance (ANOVA) (diet condition $\times$ surgery condition) was conducted to determine the difference in the number of caspase-3-ir and Ki-67-ir-labeled cells in the granule cell layer of the DG, CA1 region of the hippocampus of the ipsilesional hemisphere, in the thalamic region of the ipsilesional hemisphere, contralesional hemisphere, and whole-brain Western blot protein expression of caspase-3. Post hoc Tukey's tests were used to identify group differences. One-way ANOVA was conducted to determine the difference in number of caspase-3-ir-labeled and Ki-67 -ir-labeled cells between the ipsilesional and contralesional hemispheres, within the MCAo surgery condition. Pearson's correlation was conducted to explore correlations between infarct size and the number of caspase-3-ir and Ki-67ir-labeled cells in the thalamic region of the ipsilesional hemisphere and contralesional hemisphere; and correlations between the number of caspase-3-ir-labeled cells and Ki-67 -ir-labeled cells. Spearman correlation tests were conducted to detect correlations between the number of caspase-3-ir and Ki-67-ir-labeled cells in brain regions of interest (ipsilesional CA1 region, ipsilesional DG, and ipsilesional thalamic region) and the behavioral outcomes. The accepted $P$-value for all analysis was 0.05 .

\section{Results}

\section{Infarct analysis}

In MCAo-operated animals, right hemisphere infarct was seen in cortical and subcortical tissue, as depicted in Figure 1. Variability was seen in infarct size. In the thalamic region of the ipsilesional hemisphere, the number of caspase3 -ir cells correlated with infarct size $\left(r^{2}=0.62, P<0.01\right)$ and
Ki-67-ir-labeled cells $\left(r^{2}=0.69, P<0.01\right)$. The number of caspase-3-ir and $\mathrm{Ki}-67$-ir-labeled cells in the thalamic region of the ipsilesional hemisphere positively correlated with each other $\left(r^{2}=0.78, P<0.01\right)$. Infarct size did not correlate with the number of caspase-3-ir and Ki-67-ir-labeled cells in the thalamic region of the contralesional hemisphere.

\section{Immunohistochemistry of the}

hippocampus of the ipsilesional hemisphere No effects for surgery or diet condition on caspase-3-irlabeled cells were seen in the DG or CA1 region of the hippocampus of the ipsilesional hemisphere at 6 weeks after surgery (for CA1 surgery, $F[1,22]=1.24, P=0.28$; CA1 diet, $F[1,22]=0.08, P=0.78$; DG surgery, $F[1,21]=3.50$, $P=0.08$; and for DG diet, $F[1,21]=2.12, P=0.16$ ). No effects of surgery or diet condition on Ki-67-ir-labeled cells were seen in the DG or CA1 region of the hippocampus of the ipsilesional hemisphere at 6 weeks after surgery (CA1 surgery, $F[1,22]=2.62, P=0.12$; CA1 diet, $F[1,22]=0.37$, $P=0.55$; DG surgery, $F[1,21]=0.38, P=0.54$; and $\mathrm{DG}$ diet $F[1,21]=0.30, P=0.59)$.

\section{Immunohistochemistry of the thalamic region of the ipsilesional hemisphere}

MCAo-operated animals showed greater expression of caspase-3-ir-labeled cells than did sham-operated animals $(F[1,14]=37.92, P<0.01)$ (MCAo mean $[\mathrm{M}]=2,859$, standard error of the mean $[\mathrm{SEM}]=382$; sham $\mathrm{M}=148$, $\mathrm{SEM}=28$ ) (Figure 2). Similarly, MCAo-operated animals showed greater expression of Ki-67-ir-labeled cells than did sham-operated animals $(F[1,14]=17.68, P<0.01)$ (MCAo
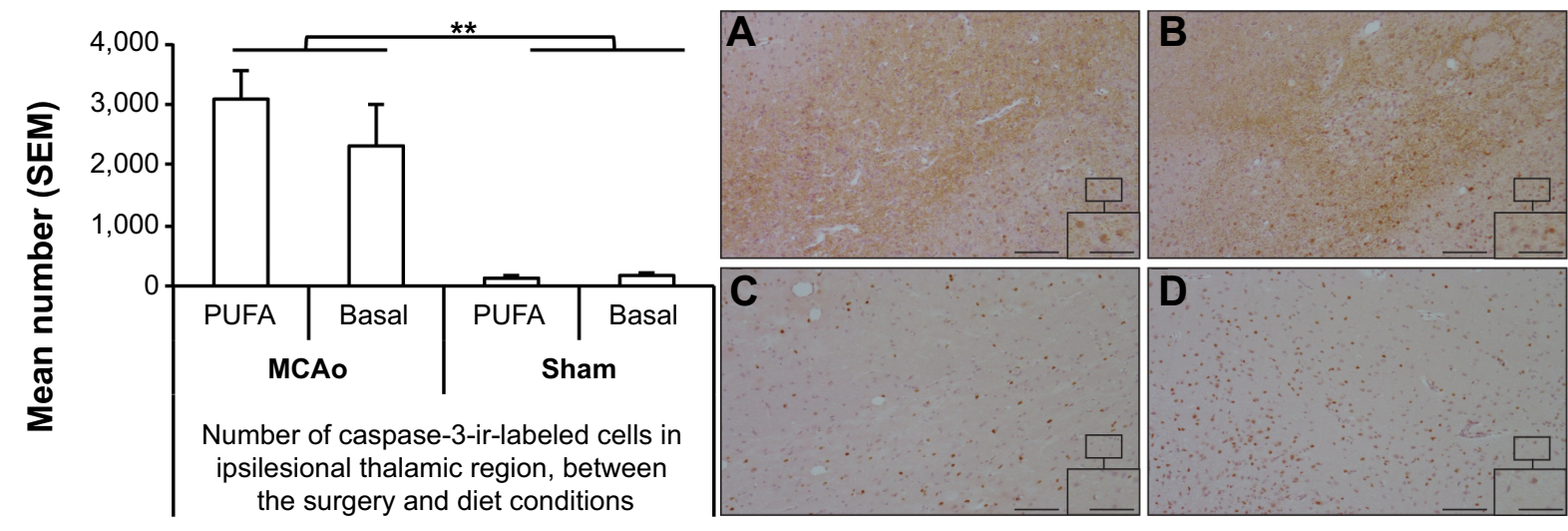

Figure 2 Mean number $( \pm$ SEM) and immunoreactive staining of caspase-3-ir cells between surgery and diet conditions in ipsilesional thalamic region at 6 weeks postsurgery. Notes: Sham = Sham surgery condition; Basal = Basal diet-fed rats; PUFA = polyunsaturated fatty acid supplemented rats. (A) Ipsilesional thalamic region of a n-3-LC-PUFA diet and MCAo surgery-affected rat. (B) Ipsilesional thalamic region of a basal diet and MCAo surgery-affected rat. (C) Ipsilesional thalamic region of a n-3-LC-PUFA diet and sham surgery-affected rat. (D) Ipsilesional thalamic region of a basal diet and sham surgery-affected rat. A I0× magnification rostral section; scale bar I00 $\mu$ m. Right-hand corner inset: 40× magnification; scale bar $25 \mu \mathrm{m}$. Bregma $-3.60 \mathrm{~mm}$. $\mathrm{n}=6$ per experimental group. **P-value significant at $0.0 \mathrm{I}$.

Abbreviations: caspase-3-ir, caspase-3-like immunoreactivity; MCAo, middle cerebral artery occlusion; n-3-LC-PUFA, long-chain omega-3 polyunsaturated fatty acid; SEM, standard error of the mean. 
$\mathrm{M}=17.4, \mathrm{SEM}=3.7$; sham $\mathrm{M}=1.2$, SEM =0.19) (Figure 3). No differences were seen between dietary groups. No interaction effects were seen.

\section{Immunohistochemistry of the thalamic region of the contralesional hemisphere}

ANOVA showed a significant effect of surgery condition on the number of caspase-3-ir-labeled cells ( $F$ $[1,22]=15.46, P<0.01$ ), with MCAo-operated animals showing more caspase-3-ir-labeled cells (caspase-3, MCAo $\mathrm{M}=254$, SEM $=31$; sham $\mathrm{M}=86$, $\mathrm{SEM}=28$ ). A significant effect of diet was also found $(F[1,22]=7.64, P<0.05)$. Animals supplemented with n-3-LC-PUFA showed fewer caspase3-ir-labeled cells ( $\mathrm{n}-3$-LC-PUFA, $\mathrm{M}=107$, SEM =17; basal, $M=218, S E M=55$ ), as depicted in Figure 4 . No effect of surgery condition was seen on the number of Ki-67-ir-labeled cells $(F[1,22]=2.25, P=1.51)$. A significant effect of diet condition was found $(F[1,22]=9.72, P<0.01)$. Animals supplemented with n-3-LC-PUFA showed more Ki-67-ir-labeled cells (Ki-67, n-3LC-PUFA, $\mathrm{M}=2.6, \mathrm{SEM}=0.29$; basal, $\mathrm{M}=1.2, \mathrm{SEM}=0.32$ ). No interaction effects were seen, as depicted in Figure 5.

\section{Immunohistochemistry of the ipsilesional} hemisphere compared with contralesional hemisphere of middle cerebral artery occlusion-operated animals

MCAo-operated animals showed significantly more caspase3 -ir cells in the thalamic region of the ipsilesional hemisphere compared with the thalamic region of the contralesional hemisphere $(F[1,19]=45.48, P<0.01)$ (caspase-3, ipsilesional thalamic region $\mathrm{M}=2,738, \mathrm{SEM}=405$; contralesional thalamic region, $\mathrm{M}=254$, $\mathrm{SEM}=55$ ). Figure 6 shows that caspase-3-ir is morphologically consistent with the presentation of amyloid precursor protein (APP) and amyloid beta $(\mathrm{A} \beta)$ that have been previously observed, from as early as 1 week to as late as 9 months post-MCAo, in the rodent model $^{26,28,52-55}$ (that is, small, diffuse fragments and large, dense deposits that resemble plaques and that are most likely harmful for functional recovery). ${ }^{26,28,52-55}$ No differences in the number of caspase-3-ir-labeled cells were seen between dietary groups. Similarly, MCAo-operated animals showed significantly more Ki-67 in the thalamic region of the ipsilesional hemisphere compared with the thalamic region of the contralesional hemisphere $(F[1,18]=19.77, P<0.01)$ (Ki-67, ipsilesional thalamic region, $\mathrm{M}=17$, SEM $=3.7$; contralesional thalamic region, $\mathrm{M}=1.6, \mathrm{SEM}=0.33$ ). No differences were seen between dietary groups.

\section{Whole-brain Western blot expression of caspase- 3 does not differ between surgery or diet conditions at 6 weeks postsurgery}

Western blot analysis was performed to confirm the presence of caspase-3 in whole-brain tissue, across all groups of animals. Nonlocalized brain tissue expression of GAPDH did not show a significant difference between surgery $(F[1,23]=0.18, P=0.67)$ or diet conditions $(F[1,23]=0.75$, $P=0.40)$. Nonlocalized brain tissue expression of caspase-3 did not differ between surgery $(F[1,23]=1.38, P=0.25)$ or diet conditions $(F[1,23]=0.56, P=0.47$ ) (MCAo n-3-LC-PUFA, $\mathrm{M}=0.83$ [SEM=0.17]; MCAo basal, $\mathrm{M}=0.82$ [SEM=0.15];
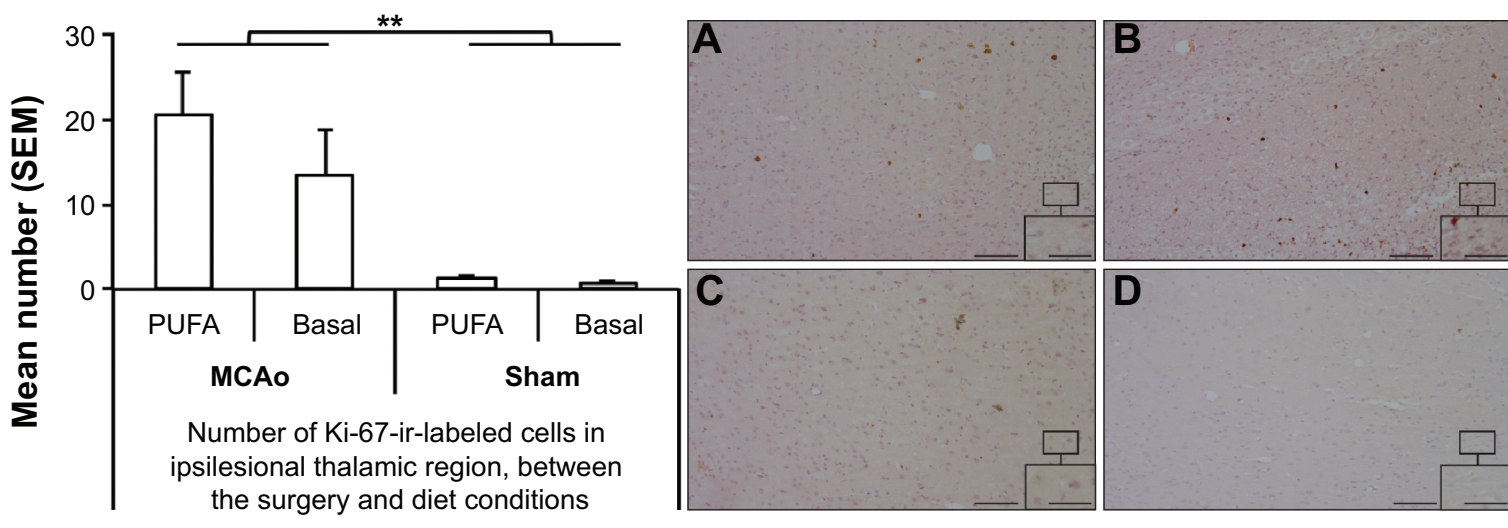

Figure 3 Mean number ( \pm SEM) and immunoreactive staining of Ki-67-ir cells between surgery and diet conditions in ipsilesional thalamic region at 6 weeks postsurgery. Notes: Sham = sham surgery condition; Basal = basal diet-fed rats; PUFA = polyunsaturated fatty acid supplemented rats. (A) Ipsilesional thalamic region of a $n-3$-LC-PUFA diet and MCAo surgery-affected rat. (B) Ipsilesional thalamic region of a basal diet and MCAo surgery-affected rat. (C) Ipsilesional thalamic region of a n-3-LC-PUFA diet and sham surgery-affected rat. (D) Ipsilesional thalamic region of a basal diet and sham surgery-affected rat. A I0× magnification rostral section; scale bar $100 \mu \mathrm{m}$. Right-hand corner inset: $40 \times$ magnification; scale bar $25 \mu \mathrm{m}$. Bregma $-3.60 \mathrm{~mm}$. $\mathrm{n}=6$ per experimental group. **P-value significant at 0.01 .

Abbreviations: Ki-67-ir, Ki-67-like immunoreactivity; MCAo, middle cerebral artery occlusion; n-3-LC-PUFA, long-chain omega-3 polyunsaturated fatty acid; SEM, standard error of the mean. 


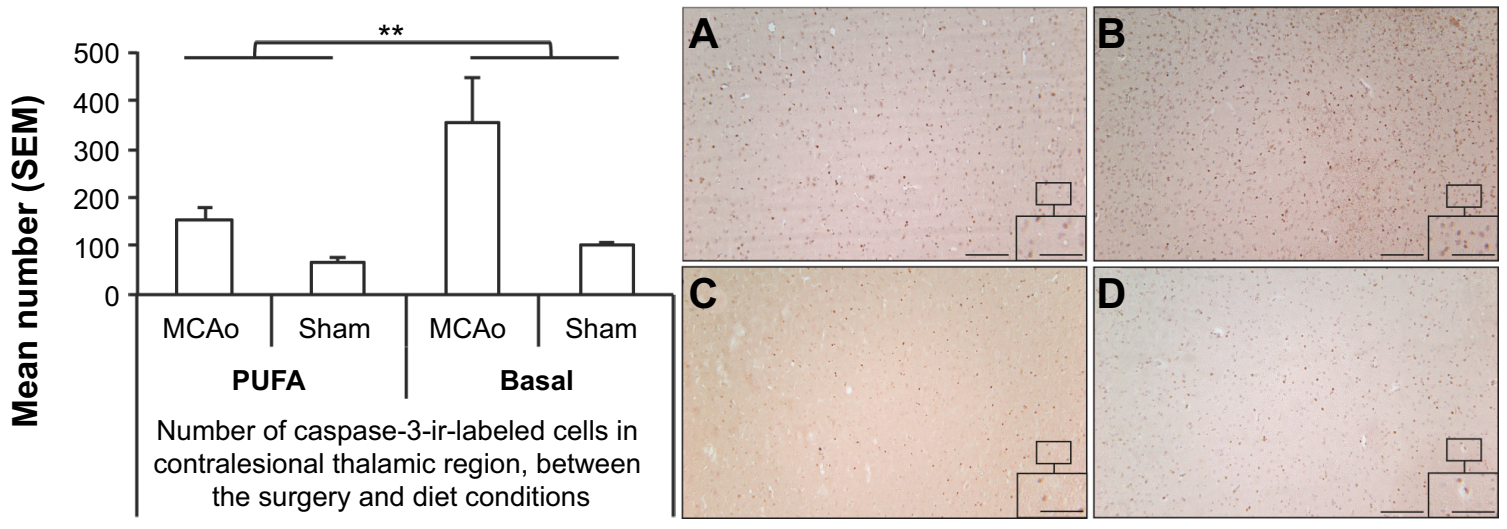

Figure 4 Mean number $( \pm$ SEM) and immunoreactive staining of caspase-3-ir cells between surgery and diet conditions in contralesional thalamic region at 6 weeks post-surgery. Notes: Sham = sham surgery condition; Basal = basal diet-fed rats; PUFA = polyunsaturated fatty acid supplemented rats. (A) Contralesional thalamic region of a n-3-LCPUFA diet and MCAo surgery-affected rat. (B) Contralesional thalamic region of a basal diet and MCAo surgery-affected rat. (C) Contralesional thalamic region of a n-3LC-PUFA diet and sham surgery-affected rat. (D) Contralesional thalamic region of a basal diet and sham surgery-affected rat. A I0× magnification rostral section; scale bar $100 \mu \mathrm{m}$. Right-hand corner inset: $40 \times$ magnification; scale bar $25 \mu \mathrm{m}$. Bregma $-3.60 \mathrm{~mm}$. $\mathrm{n}=6$ per experimental group. ${ }^{* * P} P$-value significant at $0.0 \mathrm{I}$.

Abbreviations: caspase-3-ir, caspase-3-like immunoreactivity; MCAo, middle cerebral artery occlusion; n-3-LC-PUFA, long-chain omega-3 polyunsaturated fatty acid; SEM, standard error of the mean.

sham n-3-LC-PUFA, M =0.75 [SEM=0.16]; sham basal, $\mathrm{M}=0.53[\mathrm{SEM}=0.16])$.

\section{Correlations between caspase-3 and Ki- 67 expression and behavioral outcomes at 6 weeks postsurgery}

Spearman correlations showed a positive correlation between the number of caspase-3-ir-labeled cells in the CA1 region of the hippocampus and the total emergence duration in the open field $(r h o=0.44, P<0.05)$. The number of caspase-3-ir-labeled cells in the thalamic region of the ipsilesional hemisphere positively correlated with the number of times that the rat emerged from the hide box into the open field ( $r h o=0.48, P<0.05$ ). The number of Ki-67-ir-labeled cells in the contralesional thalamic region negatively correlated with number of times the rat emerged from the hide box into the open-field arena (rho $=-0.48, P<0.05$ ). A negative correlation between the number of Ki-67-ir-labeled cells in the contralesional thalamic region and the duration of time spent moving in the open-field arena approached significance ( $r h o=-0.42, P>0.05$ ). No correlations were seen between cellular degeneration/proliferation in the DG and behavioral outcomes. No correlations were seen between novel object exploration and cellular degeneration and proliferation, in any brain region.

\section{Discussion}

We aimed to investigate the influence of n-3-LC-PUFA dietary supplementation on cell death and proliferation, at
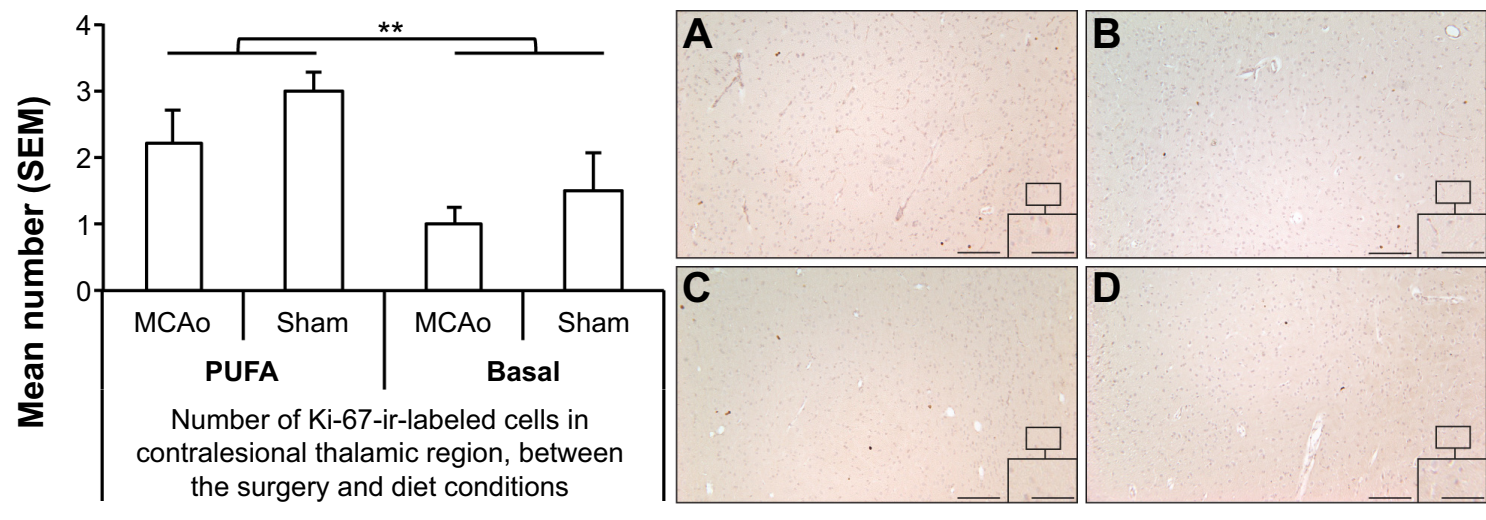

Figure 5 Mean number $( \pm$ SEM) and immunoreactive staining of Ki-67-ir cells between surgery and diet conditions in contralesional thalamic region at 6 weeks postsurgery Notes: Sham = sham surgery condition; Basal = basal diet-fed rats; PUFA = polyunsaturated fatty acid supplemented rats. (A) Contralesional thalamic region of a $n-3-L C$ PUFA diet and MCAo surgery-affected rat. (B) Contralesional thalamic region of a basal diet and MCAo surgery-affected rat. (C) Contralesional thalamic region of a n-3-LCPUFA diet and sham surgery-affected rat. (D) Contralesional thalamic region of a basal diet and sham surgery-affected rat. A $10 \times$ magnification rostral section; scale bar 100 $\mu \mathrm{m}$. Right-hand corner inset: 40x magnification; scale bar $25 \mu \mathrm{m}$. Bregma $-3.60 \mathrm{~mm}$. $\mathrm{n}=6$ per experimental group. **P-value significant at $0.0 \mathrm{I}$.

Abbreviations: Ki-67-ir, Ki-67-like immunoreactivity; MCAo, middle cerebral artery occlusion; n-3-LC-PUFA, long-chain omega-3 polyunsaturated fatty acid; SEM, standard error of the mean. 

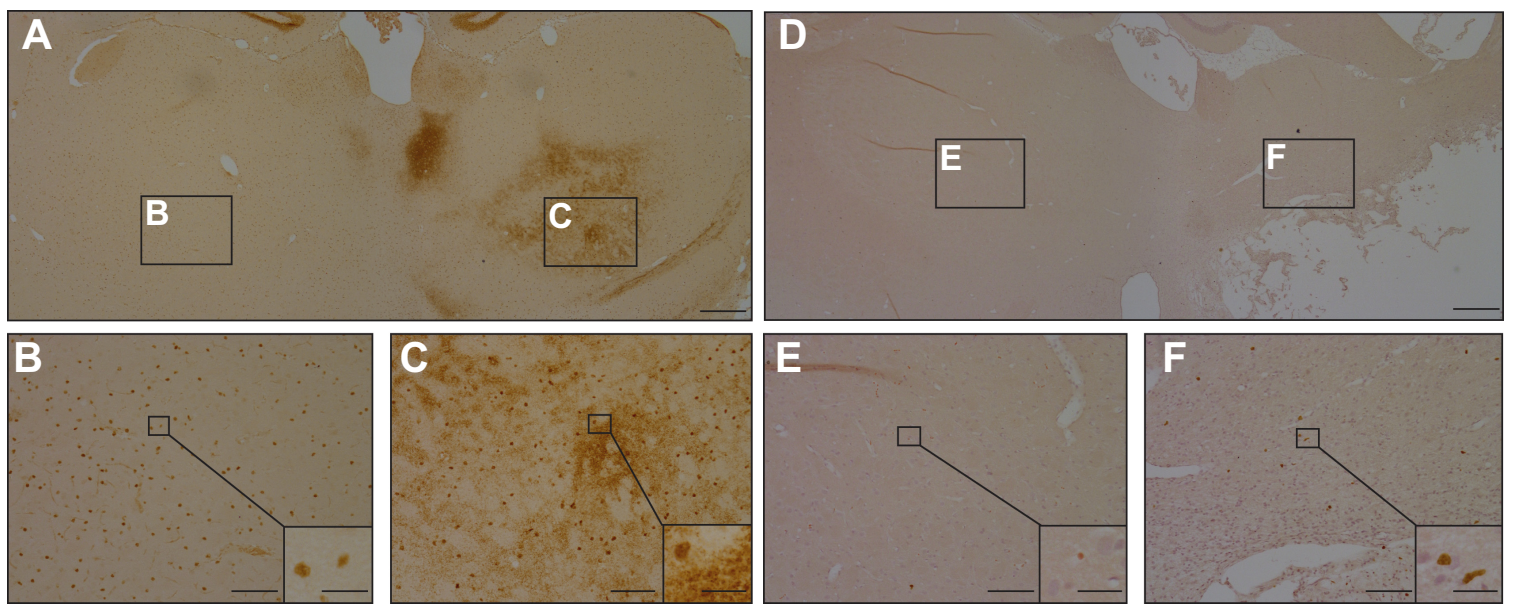

Figure 6 Caspase-3 (A) and Ki-67 (B) immunoreactive staining in bregma $-3.60 \mathrm{~mm}$ rostral section of the contralesional and ipsilesional thalamic region of a middle cerebral artery occlusion-operated rat, 6 weeks after surgery.

Notes: (A and D) At $2 \times$ magnification; scale bar $500 \mu \mathrm{m}$. (Lower $\mathbf{B}$ and $\mathbf{E}) \mathrm{A} 10 \times$ magnification of the corresponding rostral section of (A) and (D), respectively, showing the contralesional thalamic region; scale bar $100 \mu \mathrm{m}$. Right-hand corner inset: 40× magnification of the indicated portion; scale bar $25 \mu \mathrm{m}$. (Lower $\mathbf{C}$ and $\mathbf{F}$ ) A I0× magnification photograph of $(\mathbf{A})$ and $(\mathbf{D})$, respectively, showing the ipsilesional thalamic region; scale bar $100 \mu \mathrm{m}$. Right-hand corner inset: $40 \times$ magnification of the indicated portion; scale bar $25 \mu \mathrm{m}$. Pictures show bregma $-3.60 \mathrm{~mm}$. $\mathrm{n}=6$ per experimental group.

6 weeks post-MCAo, in the male hooded Wistar rat. This study also aimed to study correlations between localized cell death, and the presentation of hyperactive locomotor and anxiety-like behaviors, previously reported in these animals. ${ }^{24}$ MCAo surgery resulted in variable right-hemisphere infarct in cortical and subcortical tissue. MCAo surgery, as compared with sham surgery, was associated with both increased cellular degeneration and proliferation in the thalamic region of the ipsilesional hemisphere, which is consistent with previous research ${ }^{27,56}$ and speculated to result from retrograde degeneration of thalamocortical projections. ${ }^{57,58}$ Supplementation with anti-inflammatory n-3-LC-PUFA was associated with both increased cellular proliferation and decreased cell death in the contralesional thalamic region of MCAooperated animals, as compared with basal diet. Previous research indicates that after stroke, the brain can undergo a spatial remapping or neuroplasticity whereby contralesional undamaged brain regions are capable of taking over functions of the affected areas to some extent. ${ }^{59-62}$ Increased cellular proliferation post-MCAo in n-3-LC-PUFA-fed rats may represent a diet-assisted functional remapping.

No significant differences in caspase-3 or Ki-67-ir-labeled cells were seen in the CA1 region and DG of the ipsilesional hemisphere, between surgery and diet conditions. It is likely that the transient ischemia-associated cell degeneration and subsequent proliferation was predominantly completed by 6 weeks postsurgery, when the histoimmunological analysis was conducted. Indeed, earlier research suggests that CA1 neurons can completely repopulate by 1 month postinfarct in rodents. ${ }^{63}$
Not surprisingly, more localized caspase-3 and Ki67-ir cells were seen in the ipsilesional thalamic region than in the contralesional thalamic region. ${ }^{64}$ The extent of cells potentially programmed to die, as a function of retrograde cell death from the lesion, was negatively correlated to both infarct size and the number of Ki-67-ir-labeled cells. The presentation of caspase- 3 seen in the ipsilesional thalamic region is morphologically consistent with the presentation of APP and A $\beta$, previously observed poststroke in rodent models. ${ }^{55}$ However, dual immunofluorescence labeling of both caspase- 3 and APP is required to confirm this circumstantial evidence. The C-terminal cleavage product of APP is colocalized with caspase- 3 at a cellular level, ${ }^{41}$ and caspase-3 directly and efficiently cleaves to APP. ${ }^{41,65,66}$ Previous research demonstrates that one year of dietary supplementation with DHA reduces $A \beta$ deposition in the DG of non-MCAo-subjected mice. ${ }^{67}$ The influence of n-3-LC-PUFA supplementation on $\mathrm{A} \beta$ deposition poststroke has not been investigated to date.

Whole-brain biochemical expression of caspase-3 did not differ between surgery or diet conditions. This finding is inconsistent with the more specific immunohistochemistry data presented for the thalamus and hippocampus. Presumably this inconsistency reflects methodological differences, where one is averaging across whole brains as compared to specific immunohistochemical responses of the specific regions studied. It is likely that localized differences in the number of caspase-3 and Ki-67-ir-labeled cells between dietary and surgery conditions, were not large enough to result in significant differences in whole-brain tissue. Thus, in future research, it may be more valuable to study localized 
brain regions. Alternately, the lack of differences in wholebrain caspase-3 expression between groups may reflect limitations in the commonly used GAPDH housekeeping genes. Even the most widely used Western blot housekeeping genes are involved in multiple cell functions, and the expression levels of these are affected by many metabolic factors. ${ }^{68}$ The validity of the commonly used housekeeping genes as internal standards for measuring gene expression has been called into question. ${ }^{68}$ Indeed, GAPDH has been shown to overexpress and accumulate in the nucleus during cell death. ${ }^{69,70}$ In the present research, it is possible that the expression levels of GAPDH may have been confounded by earlier oxidative stress, resulting from ischemic insult, which may have obscured the experimental results.

We have previously reported that MCAo surgery is associated with more frequent emergence from a hide box into an open-field arena and more time moving around an open field compared with sham-operated rats, suggestive of stroke-related hyperactive locomotion. MCAo-operated animals spend less time interacting with a novel object, interpreted to reflect anxiety-like avoidance behavior. Animals supplemented with n-3-LC-PUFA spent less time moving around in the open-field arena than did the basal-fed rats but a higher percentage of emergence time in the center anxiety-provoking area of the open-field arena. Additionally, n-3-LC-PUFA-fed animals spent more time exploring a novel object than did basal diet fed animals, interpreted to reflect more approach and less anxiety-related avoidance behavior. ${ }^{24}$ In the present study, we found that caspase-3 expression in the CA1 region of the hippocampus correlated with time spent moving around in the open field 6 weeks postsurgery. This finding is consistent with previous research demonstrating that ischemia-induced damage to CA1 cells results in increased locomotion, in the gerbil, at 2 days following surgery. ${ }^{10,71}$ In the present study, the number of caspase-3-ir-labeled cells in the thalamic region of the ipsilesional hemisphere positively correlated with the number of times that the rat emerged from the hide box into the open field. Additionally, the number of Ki-67-ir-labeled cells in the contralesional thalamic region negatively correlated with number of times the rat emerged from the hide box into the open-field arena. These results may suggest that locomotor hyperactivity post-MCAo is associated with both hippocampus and thalamus damage. Indeed, the thalamus is essential for relay and modulation of sensory information, and processing of information necessary for sensorimotor control. ${ }^{31-35}$ Thalamic damage is known to be associated with poststroke hyperactive delirium in clinical populations. ${ }^{7}$
The behavioral assessment of the animals at 6 weeks postsurgery, a major advantage of the study, is also a major limitation as it ensured that we were unable to directly assess early cellular degeneration or the time of initiation of cellular genesis. Given that we were studying a range of behavioral outcomes in the days and weeks following surgery, administration of the commonly used proliferation marker 5-bromo-2-deoxyuridine (BrdU) was not a feasible alternative as this procedure requires multiple injections and is invasive ${ }^{72}$ and hence, likely to further distress the animal and influence the behavioral outcomes of primary interest. Furthermore, given the reduced water consumption observed in the days following surgery, administration of BrdU via animal drinking water was not a reliable option. ${ }^{73}$ Thus, we chose to use the $\mathrm{Ki}-67$ protein marker, present during all active phases of the cell cycle. ${ }^{45}$ We aimed to first establish whether persistent stroke-related cellular proliferation, in general, is correlated with the presentation of depressive/ anxiety-like and locomotor behaviors, or influenced by dietary intervention. In future research, it will be valuable to identify the type of proliferating cells, using double labelling for Ki-67 with markers for specific cell type, for example astrocytes (eg, GFAP) ${ }^{74}$ and microglia (eg, CD68). ${ }^{75}$

Previous rodent models indicate that the young and aged brain respond differently to ischemic stroke. All animals show increased cellular proliferation following brain injury; ; $^{27,56,76}$ however, aged animals show accelerated apoptosis, earlier activation of astrocytes and macrophages, and the formation of scar tissue, which appears to impair neuronal repair and recovery. ${ }^{56,76}$ Accordingly, aged animals show greater stroke impairment, infarct, and very high mortality rates, ${ }^{56,77}$ which was impractical for the study of behavioral outcomes in the present research. Thus, it is unknown whether n-3-LC-PUFA dietary supplementation influences cellular degeneration and proliferation in the aged brain.

This study is the first to demonstrate that n-3-LC-PUFA dietary supplementation influences MCAo-associated cell proliferation and degeneration in the thalamic region of the contralesional, but not in the ipsilesional hemisphere, when compared with rats fed a basal diet, prior to and for 6 weeks after surgery. Additionally, this study demonstrated that cellular degeneration and proliferation at 6 weeks postsurgery is correlated with hyperactive locomotor and anxiety-like behaviors, and is argued to reflect the locomotor and anxiety-like behaviors seen in stroke-surviving clinical populations..$^{4-11}$ It must also be noted that we have also previously identified that n-3-LC-PUFA supplementation is associated with an increased risk of hemorrhagic bleeding 
during reperfusion, in MCAo-operated rats. ${ }^{24}$ Therefore, the potential beneficial effects of n-3-LC-PUFA supplementation in populations that are at risk of hemorrhagic bleeding should be interpreted with caution. Indeed further research is required to identify any safety risks of n-3-LC-PUFA supplementation.

\section{Acknowledgments}

Special thanks to Sarah Rewell, Heidi Ho, and Kate Sidon of Florey Neuroscience Institutes, Melbourne, Australia, for assistance with experimental procedures. Special thanks to Associate Professor Giovanni M Turchini and Dr Gunveen Kaur, of Deakin University Warrnambool, Australia and the Institute of Sport Exercise and Active Living, Victoria University, Melbourne, Australia, respectively, for assistance with the analysis of the fatty acid profiles. Special thanks to Nicki Constantinou of Deakin University, Geelong Waterfront, Australia, for assistance with analysis of the behavioral data. Special thanks to Associate Professor Trevor Norman of the Department of Psychiatry, University of Melbourne, Austin Hospital, Heidelberg, Victoria, Australia, for the provision of equipment required for behavioral testing. No sources or grants contributed to the completion of this research.

\section{Author contributions}

Author contributions were as follows: MCP contributed to the design of the project, conducted the research, and wrote the manuscript; DWH, DPC, and LMC contributed to the design of the project and critically revised the manuscript; and SGC contributed to the design of the project and writing and revision of the manuscript.

\section{Disclosure}

The authors report no conflicts of interest in this work.

\section{References}

1. Mackay J, Mensah G. The Atlas of Heart Disease and Stroke. Geneva: World Health Organization; 2004.

2. Ramic M, Emerick AJ, Bollnow MR, O'Brien TE, Tsai SY, Kartje GL. Axonal plasticity is associated with motor recovery following amphetamine treatment combined with rehabilitation after brain injury in the adult rat. Brain Res. 2006;1111(1):176-186.

3. Kilic E, Kilic U, Bacigaluppi M, et al. Delayed melatonin administration promotes neuronal survival, neurogenesis and motor recovery, and attenuates hyperactivity and anxiety after mild focal cerebral ischemia in mice. J Pineal Res. 2008;45(2):142-148.

4. Bendel O, Bueters T, von Euler M, Ove Ogren S, Sandin J, von Euler G. Reappearance of hippocampal CA1 neurons after ischemia is associated with recovery of learning and memory. J Cereb Blood Flow Metab. 2005;25(12):1586-1595.

5. Duman RS. Depression: a case of neuronal life and death? Biol Psychiatry. 2004;56(3):140-145.
6. Revest JM, Dupret D, Koehl M, et al. Adult hippocampal neurogenesis is involved in anxiety-related behaviors. Mol Psychiatry. 2009;14(10):959-967.

7. McManus J, Pathansali R, Stewart R, Macdonald A, Jackson S. Delirium post-stroke. Age Ageing. 2007;36(6):613-618.

8. Bogousslavsky J, Ferrazzini M, Regli F, Assal G, Tanabe H, DelaloyeBischof A. Manic delirium and frontal-like syndrome with paramedian infarction of the right thalamus. J Neurol Neurosurg Psychiatry. 1988;51(1):116-119.

9. Perez DL, Catenaccio E, Epstein J. Confusion, hyperactive delirium, and secondary mania in right hemispheric strokes: a focused review of neuroanatomical correlates. J Neurol Neurophysiol. 2011;S1:003.

10. Kuroiwa T, Bonnekoh P, Hossmann KA. Therapeutic window of halothane anesthesia for reversal of delayed neuronal injury in gerbils: relationship to postischemic motor hyperactivity. Brain Res. 1991;563(1-2): $33-38$.

11. Wang D, Corbett D. Cerebral ischemia, locomotor activity and spatial mapping. Brain Res. 1990;533(1):78-82.

12. Fong TG, Tulebaev SR, Inouye SK. Delirium in elderly adults: diagnosis, prevention and treatment. Nat Rev Neurol. 2009;5(4):210-220.

13. Burvill PW, Johnson GA, Jamrozik KD, Anderson CS, StewartWynne EG, Chakera TM. Anxiety disorders after stroke: results from the Perth Community Stroke Study. Br J Psychiatry. 1995;166(3):328-332.

14. Aström M. Generalized anxiety disorder in stroke patients. A 3-year longitudinal study. Stroke. 1996;27(2):270-275.

15. Lindén T, Blomstrand C, Skoog I. Depressive disorders after 20 months in elderly stroke patients: a case-control study. Stroke. 2007;38(6): 1860-1863.

16. Pascoe MC, Crewther SG, Carey LM, Noonan K, Crewther DP, Linden T. Homocysteine as a potential biochemical marker for depression in elderly stroke survivors. Food Nutr Res. 2012;56:14973.

17. Gómez-Pinilla F. Brain foods: the effects of nutrients on brain function. Nat Rev Neurosci. 2008;9(7):568-578.

18. Pascoe MC, Crewther SG, Carey LM, Crewther DP. What you eat is what you are - a role for polyunsaturated fatty acids in neuroinflammation induced depression? Clin Nutr. 2011;30(4):407-415.

19. Black KL, Culp B, Madison D, Randall OS, Lands WE. The protective effects of dietary fish oil on focal cerebral infarction. Prostaglandins Med. 1979;3(5):257-268.

20. Nguemeni $C$, Delplanque $B$, Rovère $C$, et al. Dietary supplementation of alpha-linolenic acid in an enriched rapeseed oil diet protects from stroke. Pharmacol Res. 2010;61(3):226-233.

21. Blondeau N, Nguemeni C, Debruyne DN, et al. Subchronic alphalinolenic acid treatment enhances brain plasticity and exerts an antidepressant effect: a versatile potential therapy for stroke. Neuropsychopharmacology. 2009;34(12):2548-2559.

22. Bas O, Songur A, Sahin O, et al. The protective effect of fish n-3 fatty acids on cerebral ischemia in rat hippocampus. Neurochem Int. 2007; 50(3):548-554.

23. Ajami M, Eghtesadi S, Habibey R, et al. Effect of short and long-term treatment with omega-3 Fatty acids on scopolamine-induced amnesia. Iran J Pharm Res. 2012;11(2):533-540.

24. Pascoe MC, Howells DW, Crewther DP, et al. Fish oil diet associated with acute reperfusion related hemorrhage, and with reduced strokerelated sickness behaviors and motor impairment. Front Neurol. 2014;5:14.

25. Soriano MA, Ferrer I, Rodríguez-Farré E, Planas AM. Apoptosis and c-Jun in the thalamus of the rat following cortical infarction. Neuroreport. 1996;7(2):425-428.

26. Zhang J, Zhang Y, Li J, et al. Autophagosomes accumulation is associated with $\beta$-amyloid deposits and secondary damage in the thalamus after focal cortical infarction in hypertensive rats. $J$ Neurochem. 2012;120(4):564-573.

27. Northington FJ, Ferriero DM, Martin LJ. Neurodegeneration in the thalamus following neonatal hypoxia-ischemia is programmed cell death. Dev Neurosci. 2001;23(3):186-191. 
28. van Groen T, Puurunen K, Mäki HM, Sivenius J, Jolkkonen J. Transformation of diffuse beta-amyloid precursor protein and beta-amyloid deposits to plaques in the thalamus after transient occlusion of the middle cerebral artery in rats. Stroke. 2005;36(7):1551-1556.

29. Mohajerani MH, Aminoltejari K, Murphy TH. Targeted mini-strokes produce changes in interhemispheric sensory signal processing that are indicative of disinhibition within minutes. Proc Natl Acad Sci U S A. 2011;108(22):E183-E191.

30. van der Zijden JP, Bouts MJ, Wu O, et al. Manganese-enhanced MRI of brain plasticity in relation to functional recovery after experimental stroke. J Cereb Blood Flow Metab. 2008;28(4):832-840.

31. Larsen $\mathrm{KD}$, Asanuma $\mathrm{H}$. Thalamic projections to the feline motor cortex studied with horseradish peroxidase. Brain Res. 1979;172(2) 209-215.

32. Asanuma H, Hunsperger RW. Functional significance of projection from the cerebellar nuclei to the motor cortex in the cat. Brain Res. 1975;98(1):73-92.

33. Paxinos G, editor. The Rat Nervous System. 3rd ed. San Diego, CA: Elsevier Academic Press; 2004.

34. Evarts EV, Thach WT. Motor mechanisms of the CNS: cerebrocerebellar interrelations. Annu Rev Physiol. 1969;31:451-498.

35. Kurata K. Activity properties and location of neurons in the motor thalamus that project to the cortical motor areas in monkeys. $J$ Neurophysiol. 2005;94(1):550-566.

36. Schreiber SS, Baudry M. Selective neuronal vulnerability in the hippocampus - a role for gene expression? Trends Neurosci. 1995; 18(10):446-451.

37. Chen J, Nagayama T, Jin K, et al. Induction of caspase-3-like protease may mediate delayed neuronal death in the hippocampus after transient cerebral ischemia. J Neurosci. 1998;18(13):4914-4928.

38. Darsalia V, Heldmann U, Lindvall O, Kokaia Z. Stroke-induced neurogenesis in aged brain. Stroke. 2005;36(8):1790-1795.

39. Iwai M, Sato K, Omori N, et al. Three steps of neural stem cells development in gerbil dentate gyrus after transient ischemia. J Cereb Blood Flow Metab. 2002;22(4):411-419.

40. Clark RS, Kochanek PM, Watkins SC, et al. Caspase-3 mediated neuronal death after traumatic brain injury in rats. J Neurochem. 2000;74(2): 740-753.

41. Davoli MA, Fourtounis J, Tam J, et al. Immunohistochemical and biochemical assessment of caspase- 3 activation and DNA fragmentation following transient focal ischemia in the rat. Neuroscience. 2002;115(1): 125-136.

42. Beer R, Franz G, Srinivasan A, et al. Temporal profile and cell subtype distribution of activated caspase-3 following experimental traumatic brain injury. $J$ Neurochem. 2000;75(3):1264-1273.

43. Velier JJ, Ellison JA, Kikly KK, Spera PA, Barone FC, Feuerstein GZ. Caspase- 8 and caspase- 3 are expressed by different populations of cortical neurons undergoing delayed cell death after focal stroke in the rat. J Neurosci. 1999;19(14):5932-5941.

44. Bacigaluppi M, Pluchino S, Peruzzotti-Jametti L, et al. Delayed post-ischaemic neuroprotection following systemic neural stem cell transplantation involves multiple mechanisms. Brain. 2009;132(Pt 8): 2239-2251.

45. Scholzen T, Gerdes J. The Ki-67 protein: from the known and the unknown. J Cell Physiol. 2000;182(3):311-322.

46. Jones ML, Mark PJ, Mori TA, Keelan JA, Waddell BJ. Maternal dietary omega-3 fatty acid supplementation reduces placental oxidative stress and increases fetal and placental growth in the rat. Biol Reprod. 2013;88(2):37.

47. National Health and Medical Research Council. Australian Code of Practice for the Care and Use of Animals for Scientific Purposes. 7th ed. Canberra: National Health and Medical Research Council (Australia); 2004.

48. Barber RD, Harmer DW, Coleman RA, Clark BJ. 1. GAPDH as a housekeeping gene: analysis of GAPDH mRNA expression in a panel of 72 human tissues. Physiol Genomics. 2005;21(3):389-395.
49. Yu HR, Kuo HC, Huang HC, et al. Glyceraldehyde-3-phosphate dehydrogenase is a reliable internal control in Western blot analysis of leukocyte subpopulations from children. Anal Biochem. 2011;413(1): 24-29.

50. Paxinos G, Watson C. The Rat Brain in Stereotaxic Coordinates. 4th ed. London: Academic Press; 1998.

51. Svedin P, Hagberg H, Sävman K, Zhu C, Mallard C. Matrix metalloproteinase-9 gene knock-out protects the immature brain after cerebral hypoxia-ischemia. J Neurosci. 2007;27(7):1511-1518.

52. Zhang Y, Xing S, Zhang J, et al. Reduction of $\beta$-amyloid deposits by $\gamma$-secretase inhibitor is associated with the attenuation of secondary damage in the ipsilateral thalamus and sensory functional improvement after focal cortical infarction in hypertensive rats. J Cereb Blood Flow Metab. 2011;31(2):572-579.

53. Hiltunen M, Mäkinen P, Peräniemi S, et al. Focal cerebral ischemia in rats alters APP processing and expression of Abeta peptide degrading enzymes in the thalamus. Neurobiol Dis. 2009;35(1):103-113.

54. Mäkinen S, van Groen T, Clarke J, et al. Coaccumulation of calcium and beta-amyloid in the thalamus after transient middle cerebral artery occlusion in rats. J Cereb Blood Flow Metab. 2008;28(2):263-268.

55. Tanzi RE, Bertram L. Twenty years of the Alzheimer's disease amyloid hypothesis: a genetic perspective. Cell. 2005;120(4):545-555.

56. Popa-Wagner A, Buga AM, Kokaia Z. Perturbed cellular response to brain injury during aging. Ageing Res Rev. 2011;10(1):71-79.

57. Ross DT, Ebner FF. Thalamic retrograde degeneration following cortical injury: an excitotoxic process? Neuroscience. 1990;35(3):525-550.

58. Dihné M, Grommes C, Lutzenburg M, Witte OW, Block F. Different mechanisms of secondary neuronal damage in thalamic nuclei after focal cerebral ischemia in rats. Stroke. 2002;33(12):3006-3011.

59. Benowitz LI, Carmichael ST. Promoting axonal rewiring to improve outcome after stroke. Neurobiol Dis. 2010;37(2):259-266.

60. Dijkhuizen RM, Singhal AB, Mandeville JB, et al. Correlation between brain reorganization, ischemic damage, and neurologic status after transient focal cerebral ischemia in rats: a functional magnetic resonance imaging study. J Neurosci. 2003;23(2):510-517.

61. Takatsuru Y, Fukumoto D, Yoshitomo M, Nemoto T, Tsukada H, Nabekura J. Neuronal circuit remodeling in the contralateral cortical hemisphere during functional recovery from cerebral infarction. J Neurosci. 2009;29(32):10081-10086.

62. Carey LM, Abbott DF, Harvey MR, Puce A, Seitz RJ, Donnan GA. Relationship between touch impairment and brain activation after lesions of subcortical and cortical somatosensory regions. Neurorehabil Neural Repair. 2011;25(5):443-457.

63. Nakatomi H, Kuriu T, Okabe S, et al. Regeneration of hippocampal pyramidal neurons after ischemic brain injury by recruitment of endogenous neural progenitors. Cell. 2002;110(4):429-441.

64. Dief AEE, Jirikowski GF, Ragab KES, Ibrahim HS. Ipsilateral and contralateral cortical apoptosis in rats after unilateral middle cerebral artery occlusion. International Journal of Experimental and Clinical Anatomy. 2008; doi:10.2399/ana.08.039.

65. Milligan CE. Caspase cleavage of APP results in a cytotoxic proteolytic peptide. Nat Med. 2000;6(4):385-386.

66. Gervais FG, Xu D, Robertson GS, et al. Involvement of caspases in proteolytic cleavage of Alzheimer's amyloid-beta precursor protein and amyloidogenic A beta peptide formation. Cell. 1999;97(3):395-406.

67. Hooijmans CR, Rutters F, Dederen PJ, et al. Changes in cerebral blood volume and amyloid pathology in aged Alzheimer APP/PS1 mice on a docosahexaenoic acid (DHA) diet or cholesterol enriched Typical Western Diet (TWD). Neurobiol Dis. 2007;28(1):16-29.

68. Ferguson RE, Carroll HP, Harris A, Maher ER, Selby PJ, Banks RE. Housekeeping proteins: a preliminary study illustrating some limitations as useful references in protein expression studies. Proteomics. 2005;5(2):566-571.

69. Chuang DM, Hough C, Senatorov VV. Glyceraldehyde-3-phosphate dehydrogenase, apoptosis, and neurodegenerative diseases. Annu Rev Pharmacol Toxicol. 2005;45:269-290. 
70. Dastoor Z, Dreyer JL. Potential role of nuclear translocation of glyceraldehyde-3-phosphate dehydrogenase in apoptosis and oxidative stress. J Cell Sci. 2001;114(Pt 9):1643-1653.

71. Kuroiwa T, Bonnekoh P, Hossmann KA. Locomotor hyperactivity and hippocampal CA1 injury after transient forebrain ischemia of gerbils. Neurosci Lett. 1991;122(2):141-144.

72. Taupin P. Protocols for studying adult neurogenesis: insights and recent developments, in stem cells and regenerative medicine. Regen Med. 2007;2(1):51-62.

73. Magavi SS, Macklis JD. Identification of newborn cells by BrdU labeling and immunocytochemistry in vivo. Methods Mol Biol. 2008;438:335-343.
74. Zhu Z, Zhang Q, Yu Z, et al. Inhibiting cell cycle progression reduces reactive astrogliosis initiated by scratch injury in vitro and by cerebral ischemia in vivo. Glia. 2007;55(5):546-558.

75. Hua K, Schindler MK, McQuail JA, Forbes ME, Riddle DR. Regionally distinct responses of microglia and glial progenitor cells to whole brain irradiation in adult and aging rats. PLoS One. 2012;7(12):e52728.

76. Popa-Wagner A, Dinca I, Yalikun S, Walker L, Kroemer H, Kessler C. Accelerated delimitation of the infarct zone by capillary-derived nestinpositive cells in aged rats. Curr Neurovasc Res. 2006;3(1):3-13.

77. Lindner MD, Gribkoff VK, Donlan NA, Jones TA. Long-lasting functional disabilities in middle-aged rats with small cerebral infarcts. J Neurosci. 2003;23(34):10913-10922.

\section{Publish your work in this journal}

Neuropsychiatric Disease and Treatment is an international, peerreviewed journal of clinical therapeutics and pharmacology focusing on concise rapid reporting of clinical or pre-clinical studies on a range of neuropsychiatric and neurological disorders. This journal is indexed on PubMed Central, the 'PsycINFO' database and CAS, and is the official journal of The International Neuropsychiatric Association (INA). The manuscript management system is completely online and includes a very quick and fair peer-review system, which is all easy to use. Visit http://www.dovepress.com/testimonials.php to read real quotes from published authors.

Submit your manuscript here: http://www.dovepress.com/neuropsychiatric-disease-and-treatment-journal 